\title{
CONTROLE QUÍMICO DE PLANTAS DANINHAS NA CULTURA DO ARROZ IRRIGADO NO ESTADO DO CEARÁ ${ }^{1}$
}

\author{
FRANCISCO J. SANTOS ${ }^{2}$, JOÃO B. PITOMBEIRA ${ }^{3}$, JOÃO L. N. PINHO ${ }^{3}$ e FRANCISCO I. O. MELO ${ }^{3}$
}

\section{RESUMO}

A presente pesquisa foi conduzida, em condições de irrigação por inundação, em um solo de aluvião eutrófico da Fazenda Experimental Vale do Curu, Pentecoste-CE, em 1995. Teve como objetivo verificar a ação dos herbicidas propanil (4,80 kg/ha de i.a.), fenoxaprop-ethyl $(0,14 \mathrm{~kg} / \mathrm{ha}$ de i.a. $), \quad$ fenoxaprop-p-ethyl $(0,11 \mathrm{~kg} / \mathrm{ha}$ de i.a. $)$, oxadiazon $(0,62 \mathrm{~kg} / \mathrm{ha}$ de i.a. $)$ e as misturas: propanil + molinate $(2,52 \mathrm{~kg} / \mathrm{ha}$ de i.a. $+2,52 \mathrm{~kg} / \mathrm{ha}$ de i.a.) e propanil + 2,4-D $(2,72 \mathrm{~kg} / \mathrm{ha}$ de i.a. $+2,52 \mathrm{~kg} / \mathrm{ha}$ de i.a. $)$, no controle das plantas daninhas e efeito fitotoxico sobre as plantas das cultivares de arroz CICA-8 e METICA-1. Foi avaliado também o efeito dos herbicidas sobre a produção de arroz em casca e outros componentes da produção de grãos. Os herbicidas mais eficientes no controle das plantas daninhas foram: propanil e as misturas propanil + 2,4-D e propanil + molinate, com $90 \%$, $98 \%$ e 95\% de controle, respectivamente. Esses herbicidas proporcionaram também as maiores produções de arroz em casca, semelhantes à testemunha capinada. A altura da planta; o número de dias para o início de floração; o comprimento da panícula e o peso de 1.000 sementes, não foram afetados pelos herbicidas testados. Os sintomas de fitotoxidade causados pelos herbicidas as plantas de arroz, variaram de leve a muito leve, tendo o propanil se mostrado o mais severo, porém sem causar decréscimo significativo a produção de arroz em casca.

Palavras chaves: Oryza sativa, controle químico, irrigação.

\section{ABSTRACT}

\section{Chemical control of weeds in irrigated rice in the State of Ceará, Brazil}

A field experiment was conducted, during the dry season of 1995, on alluvial soil of the Vale do Curu Experimental Farm, State of Ceara, Brazil, in order to evaluate the effect of the herbicides: propanil (4.8 $\mathrm{kg}$ a.i.ha $\left.{ }^{-1}\right)$, fenoxapropethyl $\left(0.14 \mathrm{~kg}\right.$ a.i.ha $\left.{ }^{-1}\right)$, fenoxaprop-p-ethyl $\left(0.11 \mathrm{~kg}\right.$ a.i.ha $\left.{ }^{-1}\right)$, oxadiazon $\left(0.62 \mathrm{~kg}\right.$ a.i.ha $\left.{ }^{-1}\right)$ and the mixtures propanil + molinate $(2.52+2.52 \mathrm{~kg}$ a.i.ha $\left.{ }^{-1}\right)$ and propanil $+2,4-\mathrm{D}(2.72+0.22 \mathrm{~kg}$ a.i.ha $\left.{ }^{-1}\right)$ on the control of weeds in rice, cultivars METICA-1 and CICA-8, growing under flood irrigation. The best weed control and paddy rice yield were provided by propanil and the mixtures propanil $+2,4-\mathrm{D}$ and propanil + molinate. Plant height, days to flowering, head size and the weight of 1000 seeds were not affected by the herbicides. The symptoms of toxicity caused by the herbicides on the rice plants of the two cultivars were slightly severe.

Key words: Oryza sativa, herbicides, irrigation, toxicity.

\footnotetext{
${ }^{1}$ Recebido para publicação em 31/03/98 e na forma revisada em 25/06/99. Parte da dissertação de Mestrado do primeiro autor apresentada a CCA/UFCe para obtenção do título de Mestre em Agronomia/Fitotecnia.

${ }^{2}$ Pesquisador da Empresa de Pesquisa Agropecuária do Ceará/EPACE, CEP: 60115-221, Fortaleza/CE..

${ }^{3}$ Professores do Dept ${ }^{\circ}$ de Fitotecnia da Universidade Federal do Ceará/UFC. Fortaleza/CE.
} 


\section{INTRODUÇÃO}

O arroz é cultivado no Brasil em todas as regiões, havendo uma maior concentração nas regiões Centro-Oeste, Sul e Sudeste, responsáveis por $74 \%$ da produção nacional. O estado do Ceará participa com 1,8\% da área cultivada e 1,7\% da produção brasileira (IBGE, 1993).

No Ceará o arroz produzido provém dos plantios de sequeiro e irrigado. Em decorrência da instabilidade climática (escassez e/ou má distribuição das chuvas) as perdas de arroz nos plantios de sequeiro ocorrem com maior frequiência do que nos plantios irrigados. Neste sistema de plantio as perdas na produção são atribuídas, principalmente, ao manejo inadequado da cultura e ao alto grau de infestação das plantas daninhas nas áreas irrigadas.

Ferraz et al. (1982), Amaral \& Silveira Junior (1979), Antigua et al. (1986) entre outros, consideram as plantas daninhas como o principal fator limitante à produção do arroz, por competirem diretamente por luz, água e nutrientes, essenciais ao seu desenvolvimento.

Para Amaral \& Silveira Júnior (1979) as plantas daninhas além de competirem com o arroz, diminuindo o seu rendimento, podem prejudicá-lo de maneira indireta, agindo como hospedeiras intermediárias de pragas e doenças.

Antigua et al. (1986) consideram as perdas atribuídas às plantas daninhas ao arroz bastante variáveis, estando na dependência de uma série de fatores inerentes às espécies das populações infestantes; às cultivares de arroz utilizadas; às condições ambientais e ao manejo da cultura. Antigua \& Colon (1988) fazem referência ao Echinochloa colonum, E. cruzgalli, Ischaemum rugosum, Cyperus diformis, C. rotundus e Oryza sativa (arroz vermelho), como as espécies de plantas daninhas que mais competem com o arroz irrigado, nas principais regiões produtoras do mundo.

Andrade (1982) constatou uma redução de 16 a $80 \%$, na produtividade da cultura do arroz, cultivar Bluebelle, em função da densidade do capim-arroz (E. crusgalli).

Apesar do emprego generalizado de herbicidas nas diversas regiões orizícolas do mundo, inclusive no Ceará, a eficiência do controle está na dependência, entre outros, de fatores relacionados com o produto químico usado, clima, solo, espécies de plantas daninhas e cultivares de arroz.

$\mathrm{O}$ presente trabalho teve como objetivo avaliar a eficiência de alguns herbicidas pósemergentes, isolados ou em misturas, no controle das plantas daninhas do arroz irrigado e seus efeitos fitotóxicos sobre as plantas do arroz e alguns componentes da produção de grãos.

\section{MATERIAL E MÉTODOS}

A pesquisa foi conduzida na Fazenda Experimental Vale do Curu, em Pentecoste-CE, situada a $37^{\circ} 47^{\prime}$ latitude Sul e $39^{\circ} 16^{\prime}$ longitude Oeste, a uma altitude de 80 metros, num solo característico de um aluvião eutrófico de classificação textural franco-argiloso. A análise de fertilidade de solo revelou os seguintes resultados: $\mathrm{pH}=7,2 ; \quad \mathrm{M} . \mathrm{O}=1,55 \% ; \quad \mathrm{P}=31 \quad \mathrm{mg} / \mathrm{cm}^{3}$; $\mathrm{K}=182 \mathrm{mg} / \mathrm{cm}^{3} ; \quad \mathrm{Ca}^{++}=10,7 \quad$ meq $/ 100 \mathrm{~cm}^{3} \quad$ e $\mathrm{Al}^{+++}=0,0$ meq $/ 100 \mathrm{~cm}^{3}$.

Os herbicidas testados com respectivas doses foram: propanil $(4,80 \mathrm{~kg} / \mathrm{ha}$ de i.a.), fenoxaprop-ethyl $(0,14 \mathrm{~kg} / \mathrm{ha}$ de i.a.), fenoxapropp-ethyl $(0,11 \mathrm{~kg} / \mathrm{ha}$ de i.a. $)$, oxadiazon $(0,62 \mathrm{~kg} / \mathrm{ha}$ de i.a.) e as misturas formuladas propanil + molinate $(2,52 \mathrm{~kg} / \mathrm{ha}+2,52 \mathrm{~kg} / \mathrm{ha}$ de i.a.) e propanil + 2,4-D (2,72 kg/ha+0,22 kg/ha de i.a.), todos aplicados em pós-emergência. As doses usadas para cada herbicida, representam a recomendação do fabricante, para condições semelhantes as do local do experimento.

O delineamento experimental empregado foi o de blocos ao acaso com quatro repetições com arranjo dos tratamentos em parcelas subdivididas, onde as parcelas foram constituídas pelos herbicidas e as sub-parcelas pelas cultivares CICA-8 e METICA-1. Dois tratamentos 
testemunha foram adicionados, sendo um com controle sistemático das plantas daninhas à enxada durante todo o ciclo da cultura e um outro sem nenhum controle.

Cada sub-parcela continha 12 fileiras de 5,0 m de comprimento e área útil formada pelas oito fileiras centrais com a eliminação de $0,50 \mathrm{~m}$ nas suas extremidades, totalizando $8,0 \mathrm{~m}^{2}$. O preparo do solo da área experimental consistiu de uma aração seguida de duas gradagens. O plantio foi efetuado em sulcos abertos com enxadas, distanciados de $0,25 \mathrm{~m}$, onde colocou-se 100 sementes por metro de sulco.

Foram realizadas duas adubações nitrogenadas em cobertura, aos 27 e 65 dias após o plantio, coincidindo com o início de perfilhamento do arroz e diferenciação do primórdio floral, respectivamente. Em cada ocasião foram aplicados, a lanço, sobre a lâmina d'água, $45 \mathrm{~kg}$ de N/ha na forma de uréia.

A irrigação inicial ocorreu logo após o plantio, para permitir a germinação das sementes de arroz. A formação da lâmina d'água permanente foi iniciada três dias após a aplicação dos herbicidas, mantendo-se até 10 dias antes da colheita.

Os herbicidas foram aplicados 21 dias após o plantio, quando o arroz se encontrava no estádio de duas a três folhas e as plantas daninhas no estádio de quatro ou mais folhas. Utilizou-se um pulverizador costal com pressão constante de $40 \mathrm{lb} /$ pol$^{2}$, gerada por $\mathrm{C}_{2}$, equipado com quatro bicos com pontas de jatos em leque (Teejet 80.03 ), espaçados de $50 \mathrm{~cm}$ entre si e vazão de 300 litros/ha.

As avaliações realizadas na cultura do arroz foram: fitotoxicidade aos herbicidas, início da floração, altura da planta, comprimento da panícula, peso de 1.000 sementes e produção de grãos em casca. As avaliações do controle das plantas daninhas foram visuais e realizadas aos 7 , 21 e 28 dias após a aplicação dos herbicidas (DAA), utilizando-se o método do European
Weed Research Council-EWRC adaptado por EMBRAPA (1977).

O mesmo procedimento foi adotado nas avaliações do efeito fitotóxico dos herbicidas ao arroz, aos 7 e 14 dias após a aplicação. As notas variaram de 1 a 9 , sendo 1 para ausência de fitoxicidade e 9 para morte total das plantas.

Os dias para inicio da floração do arroz foram considerados como os decorrentes, entre o plantio e a emergência de $50 \%$ das panículas. A altura das plantas, realizada no momento da colheita, foi obtida de dez plantas tomadas aleatoriamente em cada sub-parcela, medida do nível do solo à extremidade da panícula do colmo principal. No comprimento da panícula considerou-se a distância entre o nó inferior à extremidade da última espigueta.

A produção de grãos em casca da área útil $\left(8,0 \mathrm{~m}^{2}\right)$ de cada subparcela foi determinada após a secagem ao sol e retirada das impurezas, com a umidade corrigida para $13 \%$.

A determinação do peso de 1.000 sementes obedeceu às "Regras para Análise de Sementes" estabelecidas pelo Ministério da Agricultura e Reforma Agrária (Brasil, 1992).

A análise estatística dos dados foi realizada em microcomputador 486 DX4-100, utilizando-se o módulo de modelos lineares do Software NTIA desenvolvido pela EMBRAPA. As notas atribuídas ao controle das plantas daninhas e fitotoxicidade foram transformadas para $\sqrt{x}$, após constatação de melhor ajustamento à normalização dos dados.

\section{RESULTADOS E DISCUSSÃO}

Os herbicidas mais eficientes no controle das plantas daninhas (Tabela 1) foram o propanil $\mathrm{e}$ as misturas formuladas propanil + molinate $\mathrm{e}$ propanil + 2,4-D, alcançando aos 28 dias após a aplicação, níveis de controle de 95\%, 90\% e 98\%, respectivamente (Tabela 2). Resultados semelhantes foram obtidos por Colon et al. (1990) e Antigua et al. (1990). 
TABELA 1. População inicial de plantas daninhas na área experimental.

\begin{tabular}{lcl}
\hline Famílias & $\%$ & Espécies \\
\hline Poáceas & 35,6 & $\begin{array}{l}\text { Ischaemum rugosum, Echinochloa crusgalli, Brachiaria sp., Panicum rivulare } \\
\text { and Panicum sp. }\end{array}$ \\
Ciperáceas & 59,3 & $\begin{array}{l}\text { Cyperus rotundus e Cyperus esculentus } \\
\text { Aeschynomene rudis, Macroptilium lathyroides, Crotalaria retusa, Ipomaea } \\
\text { Outras }\end{array}$ \\
\hline
\end{tabular}

TABELA 2. Controle das plantas daninhas aos 7, 21 e 28 dias após a aplicação dos herbicidas (DAA), de acordo com os valores adotados pelo European Weed Research Council (EWRC), e respectivas equivalências em porcentagem.

\begin{tabular}{|c|c|c|c|c|c|c|c|}
\hline \multirow{2}{*}{ Tratamento } & \multirow{2}{*}{$\begin{array}{c}\text { Dose } \\
\text { (kg/ha i.a) }\end{array}$} & \multicolumn{2}{|c|}{$7 \mathrm{DAA}$} & \multicolumn{2}{|c|}{$21 \mathrm{DAA}$} & \multicolumn{2}{|c|}{$28 \mathrm{DAA}$} \\
\hline & & $(1-9)$ & $\%$ & $(1-9)$ & $\%$ & $(1-9)$ & $\%$ \\
\hline propanil & 4,8 & $3,16 \mathrm{bc}$ & 95 & $3,16 \mathrm{bc}$ & 95 & $2,92 \mathrm{bc}$ & 95 \\
\hline propanil + molinate & $2,52+2,52$ & $5,94 \mathrm{~b}$ & 70 & $3,68 \mathrm{~b}$ & 90 & $3,68 \mathrm{~b}$ & 90 \\
\hline propanil $+2,4-\mathrm{D}$ & $2,72+0,22$ & 1,21 & 98 & $2,22 \mathrm{~cd}$ & 98 & $1,93 \mathrm{~cd}$ & 98 \\
\hline fenoxaprop - ethyl & 0,14 & $8,23 \mathrm{a}$ & 40 & $8,23 \mathrm{a}$ & 40 & $7,45 \mathrm{a}$ & 35 \\
\hline fenoxaprop-p-ethyl & 0,11 & $8,23 \mathrm{a}$ & 40 & $7,72 \mathrm{a}$ & 30 & $7,72 \mathrm{a}$ & 30 \\
\hline oxadiazon & 0,62 & $8,46 \mathrm{a}$ & 10 & $8,67 \mathrm{a}$ & 10 & $8,45 \mathrm{a}$ & 10 \\
\hline Test. Capinada & - & 1,00 & 100 & 1,00 & 100 & 1,00 & 100 \\
\hline Test. Sem controle & - & $9,00 \mathrm{a}$ & 0 & $9,00 \mathrm{a}$ & 0 & $9,00 \mathrm{a}$ & 0 \\
\hline
\end{tabular}

- Médias seguidas pela mesma letra, na coluna, não diferem entre si pelo teste de Tukey a 5\% de probabilidade.

- Notas do EWRC variam de 1 a 9, sendo 1, controle total e 9 sem nenhum controle.

Os herbicidas fenoxaprop-ethyl, fenoxaprop-p-ethyl e oxadiazon foram pouco eficientes no controle das plantas daninhas, alcançando índices de 35, 30 e $10 \%$, respectivamente (Tabela 2). A baixa eficiência dos dois primeiros herbicidas pode ser atribuida a especificidade dos mesmos para o controle de plantas daninhas de folha estreita que resultou não atuarem sobre as folhas largas, permitindo que essas persistissem no ambiente e consequentemente interferissem na produção do arroz. Registre-se que, nas avaliações realizadas, esses herbicidas foram altamente eficientes no controle das gramíneas Echinochloa crusgalli, Ischaemum rugosum, Brachiaria sp., Panicum rivulare e Panicum sp.
A eficiência do fenoxaprop-ethyl sobre as gramíneas se compara aos resultados obtidos por Snipes \& Street (1987), Dernoeden (1988) e Oesterhuis et al. (1990).

O baixo controle exercido pelo oxadiazon pode ser atribuído ao fato de ter sido aplicado em pós-emergência, visto que esse herbicida é mais indicado para uso em pré-emergência, por agir sobre os tecidos jovens das plantas daninhas por ocasião da germinação das sementes (Adub, 1983). Uma avaliação das plantas daninhas controladas por esse herbicida mostrou que o mesmo foi eficiente no controle das plantas daninhas de folha larga Ipomoea cynanchifolia, Physalis angulata L., Crotalaria retusa e Macroptilium lathyroides. No entanto, Baker \& 
Henry (1971) citado por Lovato (1976), ao aplicar oxadiazon no início de desenvolvimento das plantas daninhas obtiveram razoável controle sobre as mesmas.

Observa-se, ainda, na Tabela 2, que a mistura propanil + 2,4-D não diferiu estatisticamente da testemunha capinada. Já o fenoxaprop-ethyl, fenoxaprop-p-ethyl e oxadiazon mostraram baixo nível de controle das plantas daninhas, não diferindo significativamente da testemunha sem controle, pelo teste de Tukey a 5\% de probabilidade. Esses resultados indicam a eficiência dos herbicidas propanil e das misturas propanil+2,4D e propanil+molinate, e pouca eficiência dos demais por não controlarem as cyperáceas que predominavam na área do ensaio. Os tratamentos propanil e propanil + molinate não diferiram estatisticamente entre si.

Os herbicidas causaram apenas sintomas, de leve a muito leve de fitotoxicidade ao arroz, sem comprometer o seu desenvolvimento (Tabela 3). Esses resultados estão de acordo com os obtidos por Smith Jr. (1961) com o propanil; Antigua et al. (1990) com a mistura de propanil + molinate e Baker \& Henry (1971) citados por Lovato (1976), com o oxadiazon.

Quanto ao fenoxaprop-ethyl, Khodayari et al. (1989), Smith Jr. (1988), Oesterhuis et al. (1990) observaram sintomas leves de fitotoxicidade, que desapareceram antes dos 14 dias após a sua aplicação.

O propanil, aos sete dias após a aplicação (DAA), manifestou sintomas de fitotoxidade ao arroz estatisticamente diferente das testemunhas com e sem capina (Tabela 3), porém não causou danos significativos a produção de arroz em casca (Tabela 4). Assim pode-se concluir que sintomas de fitotoxidade até 7 dias após aplicação do propanil não resultam em decréscimo na produção de arroz em casca.

Não houve diferença significativa entre as cultivares CICA-8 e METICA-1 quanto a fitotoxidade aos herbicidas, resultado que difere do encontrado por Snipes \& Street (1987) e Griffin \& Baker, (1990).
Os melhores rendimentos de grãos em casca foram obtidos com os tratamentos testemunha capinada $(5.670 \mathrm{~kg} / \mathrm{ha})$, propanil + 2,4-D (5.613 kg/ha), propanil (5.604 kg/ha), propanil + molinate $(5.445(\mathrm{~kg} / \mathrm{ha})$ e fenoxapropethyl (4.841 $\mathrm{kg} / \mathrm{ha})$, que não diferiram estatisticamente entre si ao nível de $5 \%$ de probabilidade pelo teste de Tukey (Tabela 4).

Os tratamentos com oxadizon e fenoxaprop-p-ethyl proporcionaram os mais baixos níveis de produtividade, $3.854 \mathrm{~kg} / \mathrm{ha}$ e $3.738 \mathrm{~kg} / \mathrm{ha}$, respectivamente, estatisticamente semelhante ao da testemunha sem controle ( $3.558 \mathrm{~kg} / \mathrm{ha})$.

Resultados similares, utilizando propanil e propanil + molinate em arroz irrigado, foram obtidos por Antigua et al. (1990) durante os anos de 1988 e 1989 em trabalhos conduzidos no Instituto de Pesquisa do Arroz na Colômbia, quando os autores obtiveram os mais altos níveis de produtividades com propanil (3,6 kg/ha de i.a.) e a mistura propanil + molinate $(2,16 \mathrm{~kg} / \mathrm{ha}$ de i.a. $+2,16 \mathrm{~kg} / \mathrm{ha}$ de i.a.).

A cultivar METICA-1 com $4.996 \mathrm{~kg} / \mathrm{ha}$ alcançou produtividade superior a cv. CICA-8 $(4.585 \mathrm{~kg} / \mathrm{ha})$, sendo esta diferença significativa ao nível de 5\% de probabilidade (Tabela 4). Resultados similares foram obtidos nos trabalhos de Santos et al. (1992a, 1992b e 1993), conduzidos nos municípios de Iguatu e BarbalhaCE.

$\mathrm{O}$ peso de 1.000 sementes não foi influenciado pelos herbicidas aplicados, nem pela infestação das plantas daninhas, uma vez que não houve diferenças significativas entre os tratamentos com herbicidas e as testemunhas com capina e sem capina (Tabela 4). Antigua et al. (1986) e Blanco et al. (1991), constataram que a espécie Echinochloa crusgalli não influenciou o peso de 1.000 sementes.

A cultivar METICA-1 apresentou peso de 1.000 sementes superior a cv. CICA-8, diferindo estatisticamente ao nível de 5\% de probabilidade (Tabela 5). 
TABELA 3. Fitotoxicidade dos herbicidas às cultivares de arroz CICA-8 e METICA-1, aos 7 e 14 dias após a aplicação (DAA), de acordo com os valores adotados pelo European Weed Research Council (EWRC).

\begin{tabular}{|c|c|c|c|c|c|c|c|}
\hline \multirow[t]{2}{*}{ Tratamento } & \multirow{2}{*}{$\begin{array}{c}\text { Dose } \\
\text { (kg/ha i.a) }\end{array}$} & \multicolumn{3}{|c|}{7 DAA } & \multicolumn{3}{|c|}{14 DAA } \\
\hline & & CICA-8 & METICA-1 & MÉDIA & CICA-8 & METICA-1 & MÉDIA \\
\hline propanil & 4,8 & 2,46 & 2,72 & $2,59 \mathrm{a}$ & 1,42 & 1,51 & $1,66 \mathrm{a}$ \\
\hline propanil+molinate & $2,52+2,52$ & 1,71 & 2,72 & $1,96 \mathrm{ab}$ & 1,21 & 1,76 & $1,48 \mathrm{a}$ \\
\hline propanil+2,4-D & $2,72+0,22$ & 1,98 & 1,44 & $1,71 \mathrm{ab}$ & 1,00 & 1,00 & $1,00 \mathrm{a}$ \\
\hline fenoxaprop-ethyl & 0,14 & 1,71 & 1,21 & $1,44 \mathrm{ab}$ & 1,00 & 1,00 & $1,00 \mathrm{a}$ \\
\hline fenoxaprop-p-ethyl & 0,11 & 1,63 & 1,44 & $1,53 \mathrm{ab}$ & 1,00 & 1,00 & $1,00 \mathrm{a}$ \\
\hline oxadiazon & 0,62 & 1,93 & 1,93 & $1,93 a b$ & 1,00 & 1,00 & $1,00 \mathrm{a}$ \\
\hline Test. c/ capina & - & 1,00 & 1,00 & $1,00 \mathrm{~b}$ & 1,00 & 1,00 & $1,00 \mathrm{a}$ \\
\hline Test. s/ capina & - & 1,00 & 1,00 & $1,00 \quad \mathrm{~b}$ & 1,00 & 1,00 & $1,00 \mathrm{a}$ \\
\hline Média & - & $1,67 \mathrm{~A}$ & $1,62 \mathrm{~A}$ & - & $1,07 \mathrm{~B}$ & $1,15 \mathrm{~B}$ & - \\
\hline
\end{tabular}

TABELA 4. Efeito dos herbicidas sobre o rendimento de grãos de arroz em casca ( $\mathrm{kg} / \mathrm{ha})$ das cultivares CICA-8 e METICA-1.

\begin{tabular}{lcccc}
\hline \multicolumn{1}{c}{ Tratamento } & $\begin{array}{c}\text { Dose } \\
\text { kg/ha i. a. })\end{array}$ & CICA-8 & METICA-1 & Média \\
\hline propanil & 4,8 & 5.354 & 5.854 & $5.604 \mathrm{a}$ \\
propanil+molinate & $2,52+2,52$ & 5.202 & 5.689 & $5.445 \mathrm{a}$ \\
propanil+2,4-D & $2,72+0,22$ & 5.366 & 5.859 & $5.613 \mathrm{a}$ \\
fenoxaprop-ethyl & 0,14 & 4.791 & 4.891 & $4.841 \mathrm{a}$ \\
fenoxaprop-p-ethyl & 0,11 & 3.547 & 3.928 & $3.738 \mathrm{~b}$ \\
oxadiazon & 0,62 & 3.591 & 4.117 & $3.854 \mathrm{~b}$ \\
Test. c/ capina & - & 5.490 & 5.850 & $5.670 \mathrm{a}$ \\
Test. sem capina & - & 3.337 & 3.779 & $3.558 \mathrm{~b}$ \\
\hline Média & - & $4.585 \mathrm{~B}$ & $4.996 \mathrm{~A}$ & - \\
\hline
\end{tabular}

- Médias em uma mesma coluna seguidas de mesma letra minúscula ou na linha seguida de mesma letra maiúscula não diferem entre si pelo teste de Tukey a $5 \%$ de probabilidade.

$\mathrm{Na}$ cultivar METICA-1 a altura das plantas atingiu $83,46 \mathrm{~cm}$ e o comprimento da panícula $19,90 \mathrm{~cm}$, estatisticamente superior a cv. CICA-8, com $76 \mathrm{~cm}$ de altura e $18,21 \mathrm{~cm}$ de comprimento da panícula, ao nível de $5 \%$ pelo teste de Tukey (Tabela 6). Com relação ao número de dias para floração a cv. METICA-1 mostrou-se mais precoce, iniciando a floração aos 97 dias, enquanto que a cv. CICA- 8 atingiu essa fase aos 102 dias.

Vale salientar que Santos et al. (1992a,1992b e 1993) obtiveram resultados similares com relação a altura de planta, comprimento de panícula e número de dias para floração das cultivares CICA-8 e METICA-1.

A não significância em altura das plantas entre os tratamentos, contraria os resultados obtidos por Antigua et al. (1986) que encontraram redução no porte das plantas de arroz quando cresceram junto com as plantas daninhas. Segundo Antigua \& Colon, (1988) esses dados contraditórios em pesquisas envolvendo a competição de plantas daninhas com arroz é normal, pois a redução do porte do arroz é função das espécies da população infestantes e da densidade populacional, tanto da planta do arroz como das plantas invasoras. 
TABELA 5. Efeito dos herbicidas sobre o peso de 1.000 sementes das cultivares de arroz CICA- 8 e METICA-1.

\begin{tabular}{lcccc}
\hline \multirow{2}{*}{ Tratamentos } & $\begin{array}{c}\text { Dose } \\
\text { (kg/ha i. a.) }\end{array}$ & \multicolumn{3}{c}{ Peso de 1.000 sementes (g) } \\
\cline { 2 - 5 } propanil & 4,8 & 24,81 & 26,57 & Média \\
propanil+molinate & $2,52+2,52$ & 24,00 & 26,46 & $25,69 \mathrm{a}$ \\
propanil+2,4-D & $2,72+0,22$ & 24,64 & 26,68 & $25,66 \mathrm{a}$ \\
fenoxaprop-ethyl & 0,14 & 24,54 & 26,08 & $25,31 \mathrm{a}$ \\
fenoxaprop-p-ethyl & 0,11 & 24,53 & 26,34 & $25,43 \mathrm{a}$ \\
oxadiazon & 0,62 & 24,46 & 26,55 & $25,51 \mathrm{a}$ \\
Test. c/ capina & - & 24,06 & 26,53 & $25,58 \mathrm{a}$ \\
Test. s/ capina & - & 24,48 & 26,53 & $25,58 \mathrm{a}$ \\
\hline Média & - & $24,56 \mathrm{~B}$ & 26,45 A & - \\
\hline - Médias em uma mesma coluna seguidas de mesma letra minúscula ou na linha seguida de mesma letra maíscula não diferem \\
entre si pelo teste de Tukey a 5\% de probabilidade.
\end{tabular}

TABELA 6. Efeito dos herbicidas sobre a altura da planta, comprimento da panícula e dias para início da floração das cultivares de arroz CICA-8 e METICA-1.

\begin{tabular}{|c|c|c|c|c|c|c|c|c|c|}
\hline \multirow{2}{*}{ Tratamento } & \multicolumn{3}{|c|}{ Altura da Planta $(\mathrm{cm})$} & \multicolumn{3}{|c|}{ Comprimento da Panícula $(\mathrm{cm}$} & \multicolumn{3}{|c|}{ Dias para Floração } \\
\hline & Cica-8 & Metica-1 & Média & Cica- 8 & Metica-1 & Média & Cica-8 & Metica-1 & 1 Média \\
\hline propanil & 77,00 & 82,25 & $81,12 \mathrm{a}$ & 17,50 & 20,25 & $18,87 \mathrm{a}$ & 102,50 & 87,00 & $99,75 \mathrm{a}$ \\
\hline propanil + Mplonate & 76,50 & 83,00 & $79,75 \mathrm{a}$ & 18,25 & 20,00 & $19,12 \mathrm{a}$ & 102,75 & 97,75 & $100,25 \mathrm{a}$ \\
\hline propanil $+2,4-\mathrm{D}$ & 77,75 & 84,25 & $81,00 \mathrm{a}$ & 18,75 & 20,50 & $19,62 \mathrm{a}$ & 102,25 & 97,00 & $99,62 \mathrm{a}$ \\
\hline fenoxaprop-ethyl & 76,75 & 83,25 & $80,00 \mathrm{a}$ & 18,50 & 20,25 & $19,37 \mathrm{a}$ & 102,25 & 97,75 & $100,00 \mathrm{a}$ \\
\hline fenoxaprop-p-rthyl & 75,00 & 80,50 & $77,75 \mathrm{a}$ & 18,25 & 19,25 & $19,75 \mathrm{a}$ & 102,00 & 97,00 & $99,50 \mathrm{a}$ \\
\hline oxadiazon & 71,50 & 80,75 & $70,12 \mathrm{a}$ & 17,75 & 19,75 & $19,75 \mathrm{a}$ & 103,25 & 97,00 & $100,62 \mathrm{a}$ \\
\hline Testemunha c/ capina & 78,00 & 87,00 & $82,50 \mathrm{a}$ & 18,75 & 19,75 & $19,25 \mathrm{a}$ & 102,50 & 97,00 & $99,75 \mathrm{a}$ \\
\hline Test. sem capina & 75,50 & 83,75 & $79,62 \mathrm{a}$ & 18,00 & 19,50 & $18,75 \mathrm{a}$ & 101,75 & 97,00 & $99,37 \mathrm{a}$ \\
\hline Médias & $76,00 \mathrm{~B}$ & $83,46 \mathrm{~A}$ & - & $18,21 \mathrm{~B}$ & $19,90 \mathrm{~A}$ & - & 102,40 & $97,31 \mathrm{~B}$ & - \\
\hline
\end{tabular}

- Médias em uma mesma coluna seguidas de mesma letra minúscula ou na mesma linha seguidas de mesma letra maiúscula, não difere entre si pelo teste de Tukey a 5\% de probabilidade.

\section{LITERATURA CITADA}

ABUD, J.K. Controle das plantas daninhas. Lavoura Arrozeira, v.37, n.347, p.18-22, 1983.

AMARAL, A.S.; SILVEIRA JUNIOR, P. Efeitos de herbicidas na emergência do arroz e controle de plantas daninhas. Lavoura Arrozeira, v.32, n.313, p.35-37, 1979.

ANDRADE, V.A. Efeito da densidade de capimarroz na produtividade de arroz irrigado.
Lavoura Arrozeira, v.35, n.335, p.30-32, 1982.

ANTIGUA, G.; COLON, C. Control integral de las malezas en el cultivo del arroz. $\mathrm{La}$ Havana: Documentacion Agropecuaria, 1988. 47p. (CIDA. Boletim Centro de information y de Reseñas, 20).

ANTIGUA, G.; COLON, C.; GARCIA, J. Utilizacion del herbicida Arrozan en el control de malezas del arroz em Cuba. Ciencia y Tecnica en la Agricultura Arroz, v.13, n.1/2, p.135-144, 1990. 
ANTIGUA, G.; COLON, C.; PALMARES, P. Efecto de la competencia de diferentes especies de las malezas en el rendimento del arroz. Ciencia y Tecnica en la Agricultura - Arroz, v.19, n.2, p.69-83, 1986.

ANUÁRIO ESTATÍSTICO DO BRASIL. Rio de Janeiro: IBGE, v.53, seção 3, p.35, 1993.

BLANCO, H.G.; AREVOLO, R.A.; CHIBA, S. et al. Efeito da convivência de Echinochloa crus-galli (L)Beauv. (capim-arroz) com plantas de arroz em diferentes níveis de nitrogênio e fósforo. Pesq. Agropec. Bras, v.26, n.2, p.193-199, fev., 1991.

BRASIL. Ministério da Agricultura. Regras para análise de sementes. 1992. 365p.

COLON, C.; ANTIGUA, G.; GARCIA, J. et. al. Mezelas de propanil com inseticidas para el controle selectivo de melezas en arroz de riego. Ciencia y Tecnica en la Agricultura - Arroz, v. 13, n.2, p.49-58, 1990.

DERNOEDEN, P.H. Fenoxaprop combined with preemergence herbicides for crabgress and goosegrass controle in turf. Hortscience, v. 23, n.1, p.154-157, 1988.

DEUBERT, R.; SAVY, F.A.; BATAGLIA, 0.C. Efeito de herbicidas no desenvolvimento e na concentração de nutrientes em amendoim (Arachis hipogaea L.). Planta Daninha, v. 1. n.1, p.19-24, 1978

EMBRAPA. Centro Nacional de Pesquisa de Arroz e Feijão. Manual de Métodos de Pesquisa em Arroz. 1977. 106p.

FERRAZ, L.C.C.B.; PITELLI, R.A.; FURLAN, V. Nematoides associados as plantas daninhas na região de Jaboticabal-SP:
Segundo relato. Planta daninha, v. 5, n.1, p.1-5, 1982.

GRIFFIN, J.L.; BAKER, J.B. Tolerance of rice (Oryza sativa) cultivares to fenoxaprop, sethoxidim and haloxifop. Weed Sci., v.38, n.6, p. 528-531, 1990.

KHODAYARI, K.; NASTASI, P.; SMITH Jr., R.J. Fenoxaprop for grass control in dry seede rice (Oriza sativa). Weed Technol., v.3, n.1, p.131-137, 1989.

LOVATO, L.A. Dois novos herbicidas para o arroz. Lavoura Arrozeira, v.29, n.293, p.13-16, 1976.

OOSTERHUIS, D.M.; WULLSCHLEGER, S.D.; HAMPTON, R.E. et al. Physiological response of rice (Oryza sativa) fenoxaprop. Weed Sci., v.38, n.6, p.459-462, 1990.

SANTOS, F.J. dos., GRANGEIRO, R.S.B., SANTOS, A.B. dos. Efeitos de doses e épocas de aplicação de nitrogênio em duas cultivares de arroz irrigado na Região Sul do Estado do Ceará. Fortaleza: EPACE, 1992a. 6p. (EPACE. Comunicado Técnico, 35).

SANTOS, F.J. dos.; GRANGEIRO, R.S.B.; SANTOS, A.B. dos. Comportamento de cultivares e linhagens de arroz irrigado no Estado do Ceará. Fortaleza: EPACE, 1992b. 8p. (EPACE. Pesquisa em Andamento, 18).

SANTOS, F.J. dos.; GRANGEIRO, R.S.B.; SANTOS, A.B. dos. METICA-1: nova cultivar de arroz irrigado para o Estado do Ceará. Fortaleza: EPACE, 1993. 4p. (EPACE. Comunicado Técnico, 39).

SMITH Jr., R.J. 3,4 - dichoropropionanilide for control of barnyardgrass in rice. Weeds, v.9, n.2, p.318-322, 1961. 
SMITH Jr., R.J. Weed thesholds in southern U.S. SNIPES, C.E.; STREET, J.E. Rice (Oryza sativa) rice Oryza sativa. Weed Tecnol., v.2, n.2, p.242-250, 1988. tolerance to fenoxaprop. Weed Sci., v.35, n.3, p.401-406, 1987. 